\title{
Impact of flucetosulfuron on weed seed bank in wet seeded rice
}

\section{S. R. Arya*}

Department of Agronomy, College of Agriculture, Vellayani, Thiruvananthapuram-695522

(Kerala), India

Elizabeth K. Syriac

Department of Agronomy, College of Agriculture, Vellayani, Thiruvananthapuram-695522 (Kerala), India

*Corresponding author. E-mail: aaryanarayan@gmail.com

\begin{abstract}
An experiment was conducted to assess the impact of flucetosulfuron, a new generation sulfonylurea herbicide, on weed seed bank dynamics of wet land paddy field. Field experiments were carried out during two consecutive seasons (Kharif 2016 and Rabi 2016-'17), to assess the bio-efficacy of flucetosulfuron in wet seeded rice which was accompanied by weed seed bank assay. Weed seed bank assay of the soil was carried out before and after the field experiments in both the seasons by the seedling emergence method. The experiment on weed seed bank was laid out in Completely Randomised Block Design (CRD) with 12 treatments replicated thrice. Flucetosulfuron @ 20, 25, and $30 \mathrm{~g} \mathrm{ha}^{-}$ applied at 2-3, 10-12, and 18-20 days after sowing (DAS) along with two control treatments viz., hand weeding at 20 and 40 DAS and unweeded control comprised the treatments. The emerging weeds were uprooted at 14 days interval up to a period of two months; categorized as grasses, broad leaved weeds, and sedges and counted. Results revealed that non-herbicidal plots recorded significantly higher count of total emerged weeds compared to herbicide applied plots. In all the herbicide applied plots, effective reduction in weed seed bank could be obtained irrespective of the dose of the herbicide. Regarding the time of application, flucetosulfuron applied at 10-12 and 18-20 DAS recorded significantly lower weed seed bank during both the seasons, compared to its application at 2-3 DAS.
\end{abstract}

Keywords: Broad leaved weeds, Flucetosulfuron, Grasses, Sedges, Weed Management, Weed Seed Bank

\section{INTRODUCTION}

The weed seed bank can be defined as the reserve of viable weed seeds present in the soil surface and are scattered throughout the soil profile (Singh et al., 2012). Weed seed bank consists of both recently shed new weed seeds and older seeds that have persisted in the soil from previous years (Menalled, 2013). According to Hossain and Begum (2015), soil seed bank is an important component of the life cycle of weeds. The soil weed seed bank is a dynamic system consisting of inputs and outputs. The inputs occur through seed rain as an outcome of effective dispersal mechanisms (wind, water, animals and human interventions) and the outputs using sprouting, predation (Chauhan et al., 2010) and seed decay or death (Mohler et al., 2012). In the rice field, many weed species occur which can produce enormous number of small seeds and vegetative propagules as an approach to subsist the pressures imposed by weed control methods (Munhoz and Felfli, 2006). Such seeds may remain on the

\section{Article Info}

DOI:10.31018/jans.v10i3.1727

Received: May 12, 2018

Revised: June 28, 2018

Accepted: July 5, 2018

\section{How to Cite}

Arya, S.R. and Syriac, E.K (2018). Impact of flucetosulfuron on Weed seed bank in Wet seeded rice. Journal of $A p-$ plied and Natural Science, 10(3): 853 - 859 soil surface or get buried after dispersal using biotic and abiotic agents, thus forming a potential seed bank which becomes the main source of weeds in rice cropping fields (Mesquita, 2017).

Weed seedbanks are the main source of weed infestation in crops, and seed bank dynamics regulate the communities of many of the most important weed species (Barberi and Lo Cascio, 2001). In rice fields, the size of the weed seedbank is highly flexible depending on the climate, relief position, soil moisture content, depth of sampling, cropping history of the areas, and management practices used by farmers (Mesquita, 2017). The size and configuration of weed seed banks and weed populaces can be reformed by the use of herbicides, planting methods, and the use of rice cultivars which are being commonly used by the farmers (Bhagat et al. 1999). According to Hossain and Begum (2015), herbicides, crop rotation, tillage, and mulching are the factors affecting size of weed seed bank.

Seed banks perform the role of solitary source of future weed populations of the both annual and 
perennial weed species, which reproduce only using seeds. (Hossain and Begum, 2015). Hence, manipulation of the weed population through the weed seed bank is an important weed management option for such weeds (Wilson et al., 1985). According to Ambrosio et al. (2004), understanding of seed bank is necessary, for representing studies of population dynamics or for establishment of weed control programmes. Therefore, understanding seed bank composition is important to develop efficient weed management strategies (Feng et al., 2015). Hossain and Begum (2015) reported that understanding fate of seeds in the seed bank can be an important component of overall weed control. Hence, weed seed bank assay can be taken as a criteria to evaluate different weed management practices and the methods which effectively reduce weed seed bank could be marked as efficient methods for weed control. It was also reported that pre-harvest applications of glyphosate can decrease seed production and impact seed viability in late flowering weeds. Nevertheless, the slow action of glyphosate indicates that weeds must be managed effectively before the plant sheds its seed near maturity.

Under direct seeded rice cultivation (DSR), weeds are the major challenge for crop growth and its effective management is inevitable in order to attain potential productivity. In large scale rice farming, herbicide-based weed management has become the smartest and most viable option because of the scarcity and high wages of labour (Anwar et al., 2012). Sulfonyl urea groups of herbicides are low dose high efficacy herbicides having acetolactase synthase (ALS) inhibition as mode of action in plants and is found to be safer for mammals. Flucetosulfuron is such a new generation herbicide having wider application window (0-25 days). Keeping the above in view, the present study has been proposed to evaluate the impact of flucetosulfuron on weed seed bank and identification of its most suitable dose and time of application for effective reduction in weed seed bank.

\section{MATERIALS AND METHODS}

The overall study was accomplished by performing two sets of experiment: Field experiment and weed seed bank assay which is based on the pot culture experiment.

Field Experiment: Field investigations were conducted during Kharif (2016) and Rabi (2016-'17) in Kalliyoor Panchayat $\left(8.4455^{\circ} \mathrm{N}\right.$ lat. and $76.9918^{\circ}$ $\mathrm{E}$ long. at an altitude of $29 \mathrm{~m}$ above mean sea level (MSL)), Nemom block, Thiruvananthapuram district, Kerala, India. A warm, humid, tropical climate was experienced in the experimental area with $32.05{ }^{\circ} \mathrm{C}$ and $23.81{ }^{\circ} \mathrm{C}$ average maximum and minimum temperature and $92.37 \%$ and 76.89 $\%$ average maximum and minimum relative hu- midity respectively. The experiment was laid out in Randomised Block Design (RBD) with 12 treatments replicated thrice viz., $\mathrm{T}_{1}$ : Flucetosulfuron @ $20 \mathrm{~g} \mathrm{ha}^{-1}$ at 2-3 DAS, $\mathrm{T}_{2}$ : Flucetosulfuron @ $25 \mathrm{~g}$ ha $^{-1}$ at 2-3 DAS, T : Flucetosulfuron @ $30 \mathrm{~g} \mathrm{ha}^{-1}$ at 2-3 DAS, $\mathrm{T}_{4}$ : Flucetosulfuron @ $20 \mathrm{~g} \mathrm{ha}^{-1}$ at 10 -12 DAS, $T_{5}$ : Flucetosulfuron @ $25 \mathrm{~g} \mathrm{ha}^{-1}$ at 10 12 DAS, $\mathrm{T}_{6}$ : Flucetosulfuron @ $30 \mathrm{~g} \mathrm{ha}^{-1}$ at $10-12$ DAS, $\mathrm{T}_{7}$ : Flucetosulfuron @ $20 \mathrm{~g} \mathrm{ha}^{-1}$ at $18-20$ DAS , $\mathrm{T}_{8}$ : Flucetosulfuron @ $25 \mathrm{~g} \mathrm{ha}^{-1}$ at $18-20$ DAS , $\mathrm{T}_{9}$ : Flucetosulfuron @ $30 \mathrm{~g} \mathrm{ha}^{-1}$ at 18-20 DAS, $\mathrm{T}_{10}$ : Bispyribac sodium @ $25 \mathrm{~g} \mathrm{ha}^{-1}$ at 15 DAS, T 11 : Hand weeding at 20 and 40 DAS and $\mathrm{T}_{12}$ : Unweeded control. The soil was Typic haplaustalf under the order Alfisols. Kanchana (PTB 50 ), a short duration rice (105 days) variety, released from Regional Agricultural Research Station, Pattambi, Kerala, India was selected as the test crop. Seeds were sown at $100 \mathrm{~kg} \mathrm{ha}^{-1}$ and crop was raised on the basis of the agronomic management practices as per Kerala Agricultural University Package of Practices Recommendations (KAU,2011). The size of the experimental plot was $5 \mathrm{~m} \times 4 \mathrm{~m}$ (gross) and $4.7 \mathrm{~m} \times 3.7 \mathrm{~m}$ (net). Flucetosulfuron and bispyribac sodium were applied on to the surface of soil using knapsack sprayer with flood jet nozzle (spray volume $500 \mathrm{~L}$ $\left.\mathrm{ha}^{-1}\right)$. The crop was fertilized with 70:35:35 $\mathrm{kg} \mathrm{ha}^{-}$ ${ }_{1}^{1}$ nitrogen $(\mathrm{N})$, phosphorus $(\mathrm{P})$, and potassium $(\mathrm{K})$ where one third $N$ and $K$, and half $P$ was applied at 15 DAS, one third $N$ and $K$, and half $P$ was applied at 35 DAS and remaining one third $\mathrm{N}$ and $\mathrm{K}$ was applied at 55 DAS. Basal dose of organic manure was supplied with well decomposed farm yard manure (FYM) with an analytical value of $0.49,0.2$, and $0.46 \% \mathrm{~N}, \mathrm{P}_{2} \mathrm{O}_{5}$, and $\mathrm{K}_{2} \mathrm{O}$ respectively. Soil was acidic in reaction ( $\mathrm{pH} 4.32)$, high in organic carbon $(0.83 \%)$, available phosphorus $\left(25.98 \mathrm{~kg} \mathrm{ha}^{-1}\right)$, and available potassium (293.96 $\left.\mathrm{kg} \mathrm{ha}^{-1}\right)$, and medium in available nitrogen (291 kg $\left.\mathrm{ha}^{-1}\right)$. At the time of sowing a thin film of water was maintained in the field and it was gradually increased to $5 \mathrm{~cm}$ at tillering and maintained till two weeks before harvest. Just before herbicide application, the field was drained, and reflooded 48 hours after application. Just before lime application also the field was drained and was repeatedly washed two days after application.

Weed seed bank assay (Pot culture experiment): Weed seed bank assay of the soil was carried out before and after the first and second crop, by the seedling emergence method (Luschei, 2003), using CRD. Soil samples were collected at a depth of $15 \mathrm{~cm}$, from each treatment, using a soil auger. Samples were cleaned of debris, larger clods were crushed and homogenized and $1 \mathrm{Kg}$ soil was transferred to germination trays of $26-\mathrm{cm}$ diameter and a depth of 4- $\mathrm{cm}$. The samples were protected from birds and other predators and entry of foreign seeds by using nets and adequate mois- 
ture condition was maintained in the seed trays. Emerging weeds were uprooted, categorized as grasses, broad leaved weeds, and sedges and weed count was taken at fortnightly intervals. At each observation, destructive sampling was done. Seed trays were further maintained and sampling procedure repeated up to 56 days.

Statistical analysis: Since the data were on weed count and weed dry weight, which showed wide variation among treatments, all the data were subjected to square root transformation and analysed using Analysis of Variance techniques (ANOVA).

\section{RESULTS AND DISCUSSION}

Weed flora composition: Grasses (Isachne miliacea, Echinochloa stagnina, E. crusgalli and E. colona), broadleaved weeds (Limnocharis flava, Commelina diffusa, Monochoria vaginalis, Marsilea quadrifolia, Ludwigia perennis, and Lindernia rotundifolia), and sedges (Schoenoplectus pungens, Cyperus haspen, C. iria and C. difformis) were the different groups of weeds emerged from the soil. Among them, broadleaved weeds contributed more to the population followed by sedges and least contribution was from grasses to the seed bank.
Grasses: Critical analysis of the data on effect of flucetosulfuron on the emergence of grasses from the soil seed bank during both the seasons (Table1 and 2) revealed that weed management practices significantly influenced the emergence of grasses. However, before the experiment, (i.e., Kharif 2016 and Rabi 2016-17), there was no significant difference among the treatments on the number of grasses emerged from the soil seed bank, at different time intervals.

After Kharif 2016, flucetosulfuron @ $25 \mathrm{~g} \mathrm{ha}^{-1}$ at 10 -12 DAS, recorded the lowest number of grasses emerged over 56 days and was found to be on par with its application @ 20 and 30 gha $^{-1}$ at 10-12 DAS and 20, 25, and $30 \mathrm{~g} \mathrm{ha}^{-1}$ at 18-20 DAS. After Rabi 2016-17, the lowest number of grasses emerged from soil seed bank was recorded by the application of flucetosulfuron @ $20 \mathrm{~g} \mathrm{ha}^{-1}$ at $18-20$ DAS which was on par with application @ $25 \mathrm{~g} \mathrm{ha}$ ${ }^{1}$ at 18-20 DAS as well as with its application @ 20, 25 , and $30 \mathrm{~g} \mathrm{ha}^{-1}$ at 10-12 DAS. Non-herbicidal plots recorded significantly higher number for emergence of grasses which was followed by the application of flucetosulfuron @ 20, 25, and $30 \mathrm{~g}$ $\mathrm{ha}^{-1}$ at 2-3 DAS along with the application of bispyribac sodium @ $15 \mathrm{~g} \mathrm{ha}^{-1}$ at 15 DAS, during both the seasons. Compared to unweeded check,

Table 1. Emergence of grasses, broadleaved weeds, and sedges from the soil seed bank, as influenced by weed management practices before and after Kharif2016.

\begin{tabular}{|c|c|c|c|c|c|c|}
\hline \multirow[t]{2}{*}{ Treatments } & \multicolumn{2}{|c|}{$\begin{array}{l}\text { Grasses } \\
\text { emerged (No./kg } \\
\text { soil in } 56 \text { days) }\end{array}$} & \multicolumn{2}{|c|}{$\begin{array}{l}\text { Broad leaved weeds } \\
\text { emerged (No./kg soil } \\
\text { in } 56 \text { days) }\end{array}$} & \multicolumn{2}{|c|}{$\begin{array}{l}\text { Sedges } \text { emerged } \\
\text { (No./kg soil in } 56 \\
\text { days) }\end{array}$} \\
\hline & $\begin{array}{l}\text { Before } \\
\text { Kharif }\end{array}$ & $\begin{array}{l}\text { After } \\
\text { Kharif }\end{array}$ & $\begin{array}{l}\text { Before } \\
\text { Kharif }\end{array}$ & $\begin{array}{l}\text { After } \\
\text { Kharif }\end{array}$ & $\begin{array}{l}\text { Before } \\
\text { Kharif }\end{array}$ & $\begin{array}{l}\text { After } \\
\text { Kharif }\end{array}$ \\
\hline $\mathrm{T}_{1}$-flucetosulfuron @ $20 \mathrm{~g} \mathrm{ha}^{-1}$ at 2-3 DAS & $\begin{array}{l}8.06 \\
(65.00)\end{array}$ & $\begin{array}{l}6.52 \\
(42.67)\end{array}$ & $\begin{array}{l}17.02 \\
(289.67)\end{array}$ & $\begin{array}{l}15.17 \\
(230.00)\end{array}$ & $\begin{array}{l}16.07 \\
(258.67)\end{array}$ & $\begin{array}{l}13.81 \\
(191.00)\end{array}$ \\
\hline $\mathrm{T}_{2}$ - flucetosulfuron @ $25 \mathrm{~g} \mathrm{ha}^{-1}$ at 2-3 DAS & $\begin{array}{l}8.13 \\
(66.33)\end{array}$ & $\begin{array}{l}6.48 \\
(42.00)\end{array}$ & $\begin{array}{l}16.43 \\
(270.67)\end{array}$ & $\begin{array}{l}15.19 \\
(231.00)\end{array}$ & $\begin{array}{l}16.38 \\
(269.00)\end{array}$ & $\begin{array}{l}13.85 \\
(192.33)\end{array}$ \\
\hline $\mathrm{T}_{3}$ - flucetosulfuron @ $30 \mathrm{~g} \mathrm{ha}^{-1}$ at 2-3 DAS & $\begin{array}{l}7.82 \\
(61.33)\end{array}$ & $\begin{array}{l}6.55 \\
(43.00)\end{array}$ & $\begin{array}{l}17.09 \\
(292.33)\end{array}$ & $\begin{array}{l}15.22 \\
(232.00)\end{array}$ & $\begin{array}{l}16.58 \\
(274.67)\end{array}$ & $\begin{array}{l}13.74 \\
(188.67)\end{array}$ \\
\hline $\mathrm{T}_{4}$ - flucetosulfuron @ $20 \mathrm{~g} \mathrm{ha}^{-1}$ at 10-12 DAS & $\begin{array}{l}7.73 \\
(60.33)\end{array}$ & $\begin{array}{l}5.23 \\
(27.33)\end{array}$ & $\begin{array}{l}16.84 \\
(284.33)\end{array}$ & $\begin{array}{l}13.42 \\
(180.00)\end{array}$ & $\begin{array}{l}16.36 \\
(268.00)\end{array}$ & $\begin{array}{l}12.18 \\
(142.33)\end{array}$ \\
\hline $\mathrm{T}_{5^{-}}$flucetosulfuron @ $25 \mathrm{~g} \mathrm{ha}^{-1}$ at 10-12 DAS & $\begin{array}{l}7.84 \\
(61.67)\end{array}$ & $\begin{array}{l}5.13 \\
(26.33)\end{array}$ & $\begin{array}{l}16.91 \\
(286.33)\end{array}$ & $\begin{array}{l}13.20 \\
(174.33)\end{array}$ & $\begin{array}{l}16.25 \\
(264.00)\end{array}$ & $\begin{array}{l}11.90 \\
(141.67)\end{array}$ \\
\hline $\mathrm{T}_{6}$ - flucetosulfuron @ $30 \mathrm{~g} \mathrm{ha}^{-1}$ at $10-12$ DAS & $\begin{array}{l}7.95 \\
(63.33)\end{array}$ & $\begin{array}{l}5.26 \\
(27.67)\end{array}$ & $\begin{array}{l}16.57 \\
(275.33)\end{array}$ & $\begin{array}{l}13.49 \\
(182.67)\end{array}$ & $\begin{array}{l}16.64 \\
(277.00)\end{array}$ & $\begin{array}{l}12.07 \\
(146.00)\end{array}$ \\
\hline $\mathrm{T}_{7}$ - flucetosulfuron @ $20 \mathrm{~g} \mathrm{ha}^{-1}$ at 18-20 DAS & $\begin{array}{l}7.62 \\
(58.33)\end{array}$ & $\begin{array}{l}5.45 \\
(29.67)\end{array}$ & $\begin{array}{l}16.96 \\
(288.00)\end{array}$ & $\begin{array}{l}13.73 \\
(188.67)\end{array}$ & $\begin{array}{l}15.74 \\
(248.33)\end{array}$ & $\begin{array}{l}11.92 \\
(142.33)\end{array}$ \\
\hline $\mathrm{T}_{8}$ - flucetosulfuron @ $25 \mathrm{~g} \mathrm{ha}^{-1}$ at 18-20 DAS & $\begin{array}{l}7.63 \\
(58.33)\end{array}$ & $\begin{array}{l}5.40 \\
(29.33)\end{array}$ & $\begin{array}{l}16.87 \\
(284.67)\end{array}$ & $\begin{array}{l}13.38 \\
(179.00)\end{array}$ & $\begin{array}{l}16.50 \\
(272.33)\end{array}$ & $\begin{array}{l}11.89 \\
(141.67)\end{array}$ \\
\hline $\mathrm{T}_{9}$ - flucetosulfuron @ $30 \mathrm{~g} \mathrm{ha}^{-1}$ at $18-20$ DAS & $\begin{array}{l}7.79 \\
(61.00)\end{array}$ & $\begin{array}{l}5.45 \\
(29.67)\end{array}$ & $\begin{array}{l}16.33 \\
(267.33)\end{array}$ & $\begin{array}{l}13.41 \\
(180.00)\end{array}$ & $\begin{array}{l}16.59 \\
(275.67)\end{array}$ & $\begin{array}{l}12.21 \\
(149.00)\end{array}$ \\
\hline $\mathrm{T}_{10}$-bispyribac sodium @ 25g ha ${ }^{-1}$ at $15 \mathrm{DAS}$ & $\begin{array}{l}7.65 \\
(59.00)\end{array}$ & $\begin{array}{l}6.50 \\
(42.33)\end{array}$ & $\begin{array}{l}17.06 \\
(291.67)\end{array}$ & $\begin{array}{l}15.18 \\
(230.33)\end{array}$ & $\begin{array}{l}15.69 \\
(246.00)\end{array}$ & $\begin{array}{l}13.33 \\
(177.67)\end{array}$ \\
\hline $\mathrm{T}_{11}$-hand weeding at 20 and $40 \mathrm{DAS}$ & $\begin{array}{l}8.29 \\
(68.67)\end{array}$ & $\begin{array}{l}7.87 \\
(62.00)\end{array}$ & $\begin{array}{l}16.76 \\
(281.33)\end{array}$ & $\begin{array}{l}16.54 \\
(273.33)\end{array}$ & $\begin{array}{l}16.15 \\
(260.67)\end{array}$ & $\begin{array}{l}14.73 \\
(217.33)\end{array}$ \\
\hline $\mathrm{T}_{12}$-unweeded control & $\begin{array}{l}8.32 \\
(69.33)\end{array}$ & $\begin{array}{l}8.18 \\
(66.67)\end{array}$ & $\begin{array}{l}17.04 \\
(290.67)\end{array}$ & $\begin{array}{l}17.72 \\
(314.33)\end{array}$ & $\begin{array}{l}16.71 \\
(279.33)\end{array}$ & $\begin{array}{l}15.35 \\
(235.67)\end{array}$ \\
\hline $\operatorname{SEm}( \pm)$ & 0.507 & 0.270 & 0.556 & 0.348 & 0.485 & 0.360 \\
\hline$C D$ & NS & 0.546 & NS & 0.714 & NS & 0.742 \\
\hline
\end{tabular}

Note: The data were subjected to square root transformation and the values given in parentheses are original values; NS-Non-significant 
Arya, S.R. and Syriac, E.K. / J. Appl. \& Nat. Sci. 10 (3): 853 - 859 (2018)

Table 2. Emergence of grasses, broadleaved weeds, and sedges from the soil seed bank, as influenced by weed management practices before and after Rabi 2016-17.

\begin{tabular}{|c|c|c|c|c|c|c|}
\hline \multirow[t]{2}{*}{ Treatments } & \multicolumn{2}{|c|}{$\begin{array}{l}\text { Grasses } \\
\text { emerged (No./kg } \\
\text { soil in } 56 \text { days) }\end{array}$} & \multicolumn{2}{|c|}{$\begin{array}{l}\text { Broad leaved weeds } \\
\text { emerged (No./kg } \\
\text { soil in } 56 \text { days) }\end{array}$} & \multirow{2}{*}{$\begin{array}{l}\text { Sedges } \\
\text { (No./kg } \\
\text { days) } \\
\text { Before } \\
\text { Rabi } \\
\end{array}$} & \multirow{2}{*}{$\begin{array}{c}\text { emerged } \\
\text { soil in } 56 \\
\text { After } \\
\text { Rabi }\end{array}$} \\
\hline & $\begin{array}{l}\text { Before } \\
\text { Rabi }\end{array}$ & $\begin{array}{l}\text { After } \\
\text { Rabi }\end{array}$ & $\begin{array}{l}\text { Before } \\
\text { Rabi }\end{array}$ & $\begin{array}{l}\text { After } \\
\text { Rabi }\end{array}$ & & \\
\hline $\mathrm{T}_{1}$-flucetosulfuron @ $20 \mathrm{~g} \mathrm{ha}^{-1}$ at 2-3 DAS & $\begin{array}{l}7.03 \\
(49.33)\end{array}$ & $\begin{array}{l}5.45 \\
(29.67)\end{array}$ & $\begin{array}{l}14.99 \\
(225.00)\end{array}$ & $\begin{array}{l}12.45 \\
(155.33)\end{array}$ & $\begin{array}{l}13.94 \\
(195.00)\end{array}$ & $\begin{array}{l}13.28 \\
(176.67)\end{array}$ \\
\hline $\mathrm{T}_{2}$ - flucetosulfuron @ $25 \mathrm{~g} \mathrm{ha}^{-1}$ at 2-3 DAS & $\begin{array}{l}7.02 \\
(49.33)\end{array}$ & $\begin{array}{l}5.31 \\
(28.33)\end{array}$ & $\begin{array}{l}14.91 \\
(222.33)\end{array}$ & $\begin{array}{l}12.12 \\
(147.33)\end{array}$ & $\begin{array}{l}14.41 \\
(207.67)\end{array}$ & $\begin{array}{l}13.36 \\
(178.33)\end{array}$ \\
\hline $\mathrm{T}_{3^{-}}$flucetosulfuron @ $30 \mathrm{~g} \mathrm{ha}^{-1}$ at 2-3 DAS & $\begin{array}{l}7.07 \\
(50.00)\end{array}$ & $\begin{array}{l}5.32 \\
(28.33)\end{array}$ & $\begin{array}{l}15.38 \\
(236.67)\end{array}$ & $\begin{array}{l}12.44 \\
(155.00)\end{array}$ & $\begin{array}{l}14.58 \\
(212.67)\end{array}$ & $\begin{array}{l}13.34 \\
(178.00)\end{array}$ \\
\hline $\mathrm{T}_{4}$ - flucetosulfuron @ $20 \mathrm{~g} \mathrm{ha}^{-1}$ at $10-12$ DAS & $\begin{array}{l}6.84 \\
(46.67)\end{array}$ & $\begin{array}{l}4.36 \\
(19.00)\end{array}$ & $\begin{array}{l}14.99 \\
(225.00)\end{array}$ & $\begin{array}{l}11.05 \\
(122.00)\end{array}$ & $\begin{array}{l}14.27 \\
(204.00)\end{array}$ & $\begin{array}{l}11.50 \\
(132.33)\end{array}$ \\
\hline $\mathrm{T}_{5^{-}}$flucetosulfuron @ $25 \mathrm{~g} \mathrm{ha}^{-1}$ at $10-12$ DAS & $\begin{array}{l}7.00 \\
(49.00)\end{array}$ & $\begin{array}{l}4.25 \\
(18.00)\end{array}$ & $\begin{array}{l}15.10 \\
(228.33)\end{array}$ & $\begin{array}{l}10.76 \\
(116.00)\end{array}$ & $\begin{array}{l}14.83 \\
(220.00)\end{array}$ & $\begin{array}{l}10.90 \\
(119.00)\end{array}$ \\
\hline $\mathrm{T}_{6}$ - flucetosulfuron @ $30 \mathrm{~g} \mathrm{ha}^{-1}$ at $10-12$ DAS & $\begin{array}{l}6.87 \\
(47.33)\end{array}$ & $\begin{array}{l}4.24 \\
(18.00)\end{array}$ & $\begin{array}{l}15.05 \\
(226.67)\end{array}$ & $\begin{array}{l}10.95 \\
(120.00)\end{array}$ & $\begin{array}{l}14.91 \\
(222.67)\end{array}$ & $\begin{array}{l}11.50 \\
(132.33)\end{array}$ \\
\hline $\mathrm{T}_{7}$ - flucetosulfuron @ $20 \mathrm{~g} \mathrm{ha}^{-1}$ at 18-20 DAS & $\begin{array}{l}6.93 \\
(48.00)\end{array}$ & $\begin{array}{l}4.03 \\
(16.33)\end{array}$ & $\begin{array}{l}14.86 \\
(221.00)\end{array}$ & $\begin{array}{l}11.07 \\
(122.67)\end{array}$ & $\begin{array}{l}14.07 \\
(198.00)\end{array}$ & $\begin{array}{l}10.97 \\
(120.67)\end{array}$ \\
\hline $\mathrm{T}_{8}$ - flucetosulfuron @ $25 \mathrm{~g} \mathrm{ha}^{-1}$ at 18-20 DAS & $\begin{array}{l}6.89 \\
(47.67)\end{array}$ & $\begin{array}{l}4.31 \\
(18.67)\end{array}$ & $\begin{array}{l}15.41 \\
(237.67)\end{array}$ & $\begin{array}{l}11.24 \\
(126.67)\end{array}$ & $\begin{array}{l}14.77 \\
(218.33)\end{array}$ & $\begin{array}{l}11.28 \\
(127.67)\end{array}$ \\
\hline $\mathrm{T}_{9}$ - flucetosulfuron @ $30 \mathrm{~g} \mathrm{ha}^{-1}$ at $18-20$ DAS & $\begin{array}{l}6.83 \\
(50.00)\end{array}$ & $\begin{array}{l}4.43 \\
(19.67)\end{array}$ & $\begin{array}{l}15.36 \\
(236.33)\end{array}$ & $\begin{array}{l}10.83 \\
(117.33)\end{array}$ & $\begin{array}{l}14.72 \\
(217.67)\end{array}$ & $\begin{array}{l}11.48 \\
(131.67)\end{array}$ \\
\hline $\mathrm{T}_{10}$-bispyribac sodium @ $25 \mathrm{~g} \mathrm{ha}^{-1}$ at $15 \mathrm{DAS}$ & $\begin{array}{l}6.99 \\
(49.00)\end{array}$ & $\begin{array}{l}5.34 \\
(28.67)\end{array}$ & $\begin{array}{l}15.35 \\
(235.67)\end{array}$ & $\begin{array}{l}12.11 \\
(146.67)\end{array}$ & $\begin{array}{l}14.43 \\
(208.33)\end{array}$ & $\begin{array}{l}13.28 \\
(176.33)\end{array}$ \\
\hline $\mathrm{T}_{11}$-hand weeding at 20 and $40 \mathrm{DAS}$ & $\begin{array}{l}7.20 \\
(52.00)\end{array}$ & $\begin{array}{l}6.41 \\
(41.00)\end{array}$ & $\begin{array}{l}15.44 \\
(238.67)\end{array}$ & $\begin{array}{l}14.38 \\
(207.00)\end{array}$ & $\begin{array}{l}14.59 \\
(213.00)\end{array}$ & $\begin{array}{l}14.46 \\
(209.00)\end{array}$ \\
\hline $\mathrm{T}_{12}$-unweeded control & $\begin{array}{l}7.24 \\
(52.33)\end{array}$ & $\begin{array}{l}6.65 \\
(44.33)\end{array}$ & $\begin{array}{l}15.47 \\
(239.33)\end{array}$ & $\begin{array}{l}14.69 \\
(216.00)\end{array}$ & $\begin{array}{l}14.52 \\
(211.00)\end{array}$ & $\begin{array}{l}15.50 \\
(240.00)\end{array}$ \\
\hline & & & & 0.397 & 0.444 & 0.407 \\
\hline$C D$ & NS & 0.392 & NS & 0.829 & NS & 0.827 \\
\hline
\end{tabular}

Note: The data were subjected to square root transformation and the values given in parentheses are original values; NS-Non-significant

the percentage reduction in grasses ranged from 55.50 to $60.51 \%$ and 55.63 to $63.16 \%$ for Kharif 2016 and Rabi 2016-17 respectively in the case of application of flucetosulfuron at 10-12 and 18-20 DAS. Compared to hand weeding treatment, the percentage reduction was 52.15 to $57.53 \%$ and 52.02 to $60.17 \%$ for Kharif 2016 and Rabi 201617 respectively. However, the reduction in emergence of grasses was found to be in the range of 29.91 to $37.80 \%$ and from 31.39 to $43.04 \%$ for Kharif 2016 and Rabi 2016-17 respectively when compared to bispyribac sodium. Kamoshita et al. (2016) stated that such difference in weed emergence between herbicidal and non-herbicidal plots indicate that the weed seed bank can increase or decrease depending on the weed management practices. Better the weed management practice, lesser will be the weed seed bank. Weed infestation without herbicide application was more severe under direct-seeding practice resulting in higher weed seed bank size. Islam (2012) also opined that the seed number and species composition of the seed bank were influenced by herbicide application.

Broadleaved weeds: Perusal of data on the emergence of broad leaved weeds from the soil seed bank, before both the seasons (Table 1 and
2), revealed that, just as in the case of grasses, there was no significant difference among the treatments on the emergence of broadleaved weeds. However, analysis of the data, after both the seasons, revealed that application of flucetosulfuron @ $25 \mathrm{~g} \mathrm{ha}^{-1}$ at 10-12 DAS recorded the lowest number of broad leaved weeds emerged from the soil which was found to be on par with its application @ 20 and $30 \mathrm{~g} \mathrm{ha}^{-1}$ at 10-12 DAS as well as with its application @ 20, 25, and $30 \mathrm{~g} \mathrm{ha}^{-1}$ at 18-20 DAS. Application of flucetosulfuron at 2-3 DAS, irrespective of its dosage resulted in significantly higher number of broadleaved weeds compared to its application at 10-12 and 18-20 DAS indicating the significance of time of application of flucetosulfuron on seed bank of broadleaved weeds.Here also, higher broadleaved weeds emergence from the soil seed bank was recorded by the non-herbicidal plots.The efficacy of flucetosulfuron applied at 10-12 and 18-20 DAS for broadleaved population reduction was assessed in comparison with unweeded check and percentage reduction of 39.98 to 44.54 and 41.36 to 46.30 was obtained for Kharif 2016 and Rabi 2016 -17 respectively. However, the reduction in population in comparison with hand weeding twice were 30.97 to $36.22 \%$ and 38.81 to $43.96 \%$ for 
Table 3. Total count of emerged weeds from the soil seed bank as influenced by weed management practices before and after Kharif2016 andRabi2016-17.

\begin{tabular}{|c|c|c|c|c|}
\hline \multirow[t]{2}{*}{ Treatments } & \multicolumn{2}{|c|}{$\begin{array}{l}\text { Total No. of weeds emerged / } \\
\mathrm{kg} \text { of soil in } 56 \text { days }\end{array}$} & \multicolumn{2}{|c|}{$\begin{array}{l}\text { Total No. of weeds emerged } \\
\mathrm{kg} \text { of soil in } 56 \text { days }\end{array}$} \\
\hline & Before Kharif & After Kharif & Before Rabi & After Rabi \\
\hline $\mathrm{T}_{1}$-flucetosulfuron @ $20 \mathrm{~g} \mathrm{ha}^{-1}$ at 2-3 DAS & $24.76(613.33)$ & $21.53(463.67)$ & $21.66(469.33)$ & $19.01(361.67)$ \\
\hline $\mathrm{T}_{2^{-}}$flucetosulfuron @ $25 \mathrm{~g} \mathrm{ha}^{-1}$ at 2-3 DAS & $24.59(606.00)$ & $21.56(465.33)$ & $21.89(479.33)$ & $18.80(354.00)$ \\
\hline $\mathrm{T}_{3}$ flucetosulfuron @ $30 \mathrm{~g} \mathrm{ha}^{-1}$ at 2-3 DAS & $25.07(628.33)$ & 21.53 (463.67) & $22.35(499.33)$ & $19.00(361.33)$ \\
\hline $\mathrm{T}_{4}$ - flucetosulfuron @ $20 \mathrm{~g} \mathrm{ha}^{-1}$ at $10-12$ DAS & $24.75(612.67)$ & $18.85(355.67)$ & $21.81(475.67)$ & $16.52(273.33)$ \\
\hline $\mathrm{T}_{5}$ - flucetosulfuron @ $25 \mathrm{~g} \mathrm{ha}^{-1}$ at $10-12$ DAS & $24.74(612.00)$ & $18.51(342.33)$ & $22.30(497.33)$ & $15.88(253.00)$ \\
\hline $\mathrm{T}_{6}$ - flucetosulfuron @ $30 \mathrm{~g} \mathrm{ha}^{-1}$ at $10-12$ DAS & $24.79(615.67)$ & $18.87(356.33)$ & $22.29(496.67)$ & $16.43(270.33)$ \\
\hline $\mathrm{T}_{7}$ - flucetosulfuron @ $20 \mathrm{~g} \mathrm{ha}^{-1}$ at $18-20$ DAS & $24.38(594.67)$ & $18.99(360.67)$ & $21.60(467.00)$ & $16.11(259.67)$ \\
\hline $\mathrm{T}_{8}$ - flucetosulfuron @ $25 \mathrm{~g} \mathrm{ha}^{-1}$ at $18-20$ DAS & $24.81(615.33)$ & $18.69(350.00)$ & $22.44(503.67)$ & $16.50(273.00)$ \\
\hline $\mathrm{T}_{9}$ - flucetosulfuron @ $30 \mathrm{~g} \mathrm{ha}^{-1}$ at $18-20$ DAS & $24.57(604.00)$ & $18.93(358.67)$ & $22.45(504.00)$ & $16.40(268.67)$ \\
\hline $\mathrm{T}_{10}$-bispyribac sodium @ $25 \mathrm{~g} \mathrm{ha}^{-1}$ at 15 DAS & $24.41(596.67)$ & $21.22(450.33)$ & $22.20(493.00)$ & $18.76(351.67)$ \\
\hline $\mathrm{T}_{11}$-hand weeding at 20 and 40 DAS & $24.72(610.67)$ & $23.51(552.67)$ & $22.44(503.67)$ & $21.38(457.00)$ \\
\hline$T_{12}$-unweeded control & $25.29(639.33)$ & $24.83(616.67)$ & $22.41(502.67)$ & $22.37(500.33)$ \\
\hline $\operatorname{SEm}( \pm)$ & 0.588 & 0.410 & 0.328 & 0.429 \\
\hline CD & NS & 0.843 & NS & 0.877 \\
\hline
\end{tabular}

Note: The data were subjected to square root transformation and the values given in parentheses are original values; NS-Non-significant

Table 4. Correlation between total weeds emerged up to 56 DAS in weed seed bank assay and total weed density and total weed dry weight at 60 DAS from the field experiment.

\begin{tabular}{|c|c|c|c|c|}
\hline \multirow[t]{2}{*}{ Parameter } & \multicolumn{2}{|c|}{$\begin{array}{l}\text { Total weed density at } 60 \text { DAS } \\
\text { in field experiment }\end{array}$} & \multicolumn{2}{|c|}{$\begin{array}{l}\text { Total weed dry weight at } 60 \\
\text { DAS in field experiment }\end{array}$} \\
\hline & Kharif (2016) & Rabi (2016-17) & Kharif (2016) & Rabi(2016-17) \\
\hline Total weeds emerged at 14 days in seed bank assay & $0.694^{\star *}$ & $0.565^{\star \star}$ & $0.718^{\star *}$ & $0.675^{\star *}$ \\
\hline Total weeds emerged at 28 days in seed bank assay & $0.642^{* *}$ & $0.564^{\star *}$ & $0.693^{* *}$ & $0.678^{* *}$ \\
\hline Total weeds emerged at 42 days in seed bank assay & $0.647^{* *}$ & $0.616^{* *}$ & $0.688^{* *}$ & $0.692^{* *}$ \\
\hline Total weeds emerged at 56 days in seed bank assay & $0.759^{* *}$ & $0.654^{\star *}$ & $0.771^{* *}$ & $0.720^{* *}$ \\
\hline $\begin{array}{l}\text { Grand total of weeds emerged over } 56 \text { days of obser- } \\
\text { vation in seed bank assay }\end{array}$ & $0.709^{* *}$ & $0.620^{* *}$ & $0.745^{\star *}$ & $0.723^{* *}$ \\
\hline
\end{tabular}

** \& * correlation is significant at 0.01 and 0.05 level respectively (2-tailed), $n=36$

Kharif 2016 and Rabi 2016-17 respectively. Compared to bispyribac sodium, the percentage reduction was18.09 to 24.31 and 13.64 to 20.91 for Kharif 2016 and Rabi 2016-17 respectively. Similar results were reported by Mesquita (2017) that herbicides are effective in reducing weed populations and hence the number of seeds added to the soil seed bank. Pereira et al. (2013) found that before the application of the herbicide treatments there was the highest number of viable weed seeds in the study area and over the application of metalachlor+diuron and diuron + pendimethalin the number of weed seeds were reduced effectively.

Sedges: Application of flucetosulfuron @ $25 \mathrm{~g} \mathrm{ha}^{-}$ ${ }^{1}$ at 18-20 DAS recorded significantly lower number of sedges emerged from the soil seed bank over a period of 56 days and was on par with its application @ 20 and $30 \mathrm{~g} \mathrm{ha}^{-1}$ at 10-12 DAS as well as with its application @ 20, 25, and $30 \mathrm{~g} \mathrm{ha}^{-1}$ at 18-20 DAS during both the seasons (Table 1 and 2). Significantly higher number of sedges emerged from the soil seed bank was reported from application of flucetosulfuron at 2-3 DAS for all the three doses. However, the highest number of sedges was recorded from unweeded control during both the crop seasons. Higher number of sedges emerged from the soil seed bank in non- herbicidal treatments compared to herbicidal treatments. Compared to unweeded control, population of sedges was reduced by 36.78 to $39.89 \%$ and 45.14 to $50.42 \%$ respectively after the Kharif 2016 and Rabi 2016-17 respectively over the application of flucetosulfuron at 10-12 and 18-20 DAS. Nevertheless, the percentage reduction was 31.44 to $34.81 \%$ and 37.00 to $43.06 \%$ respectively compared to hand weeding. Compared to bispyribac sodium 16.14 to $20.26 \%$ and 24.95 to $32.51 \%$ after Kharif 2016 and Rabi 2016-17 respectively, were the percentage population reduction. The results conform with the findings of Barberi et al. (1998), who reported that herbicides reduce weed densities and hence reduce the number of weed seeds that are produced and enter the seed bank. Vasileiadis et al. (2007) observed that in light textured soils, conventional tillage with herbicide use gradually abridged seed density over several years in the top $15 \mathrm{~cm}$ soil depth.

Total count of weeds: No significant difference was observed among the treatments in total count of emerged weeds before Kharif 2016 and Rabi 2016-17 (Table 3). However, there was considerable reduction in the total weed count before the Rabi 2016-17 compared to that of Kharif 2016, implying that the weed control measures adopted 
during Kharif 2016 was effective in reducing the weed seed bank in soil. This conforms with the findings of Barberi et al. (1998).

Critical analysis of the data on total number of weeds emerged over 56 days (Table 3 ) revealed that after Kharif 2016 and Rabi 2016-17, the lowest number of weeds was recorded from soil treated with flucetosulfuron @ $25 \mathrm{~g} \mathrm{ha}^{-1}$ at 10-12 DAS which was found to be on par with its application @ 20 and $30 \mathrm{~g} \mathrm{ha}^{-1}$ at 10-12 DAS and @ 20, 25, and $30 \mathrm{~g} \mathrm{ha}^{-1}$ at 18-20 DAS. After Rabi 2016-17, (i.e., after two seasons of the study), only 21.74 and $25.15 \%$ reduction in population was reported in unweeded control and hand weeding respectively compared to the initial weed count recorded before Kharif 2016. The percentage reduction was $41.06 \%$ in the case of control herbicide, bispyribac sodium. However, the percentage reduction in the total count of emerged weeds from soil seed bank ranged from 55.52 to $58.66 \%$ when flucetosulfuron was applied at 10-12 and 18-20 DAS compared to its application at 2-3 DAS where the percentage reduction after two seasons ranged from 41.03 to $42.49 \%$ only. These results are in conformity with the findings of Hyvolen and Salonen (2012) and Walia and Brar (2006), who opined that herbicide use reduced weed seed bank considerably.

Interestingly, in the field experiment, the treatments viz., flucetosulfuron @ 20, 25, and $30 \mathrm{~g} \mathrm{ha}$ 1 at 10-12 and 18-20 DAS recorded the lower total weed count, and the same trend was seen in the case of total weed seed bank also. Correlation (Table 4) between total weeds emerged up to 56 DAS at fortnightly interval in weed seed assay and total weed density and total weed dry weight at 60 DAS in field experiment revealed significant positive correlations. For correlation analysis, 36 pairs of observations were taken. It is evident that the weed density and weed dry weight in the field, significantly influenced the weed seed bank. Flucetosulfuron applied at 10-12 and 18-20 DAS recorded significantly lower weed dry weights during both the crop seasons compared to other weed management practices adopted. This result has directly reflected on the outcome of the weed seed bank assay.In the seed bank assay also, the lowest count of total weeds was recorded from plots where flucetosulfuron was applied at 10-12 and 18-20 DAS irrespective of their doses of application. These results are in conformity with the findings of Teasdale et al. (2004), who reported that initial weed population is directly related to the density of seeds in the seed bank. The lesser the weeds in the field, the lesser would be the seed rain. According to Hossain et al. (2014); Barros (2013) herbicides are very effective in reducing the weed populations and at the same time the number of seeds added to the soil seed bank. But, there is an exception in the case of hand weeding. In the field experiment, even though the seed density and weed dry weight of hand weeding twice treatment (T11) was comparable to the best herbicidal treatments, this effect was not manifested on the weed seed bank which is clearly evident from the results of weed seed bank assay. This could probably because unlike herbicidal treatment, the soil seed bank was unaffected by hand weeding treatment, where in only emerged weed seedlings were removed. The count of total weeds emerged from soil receiving hand weeding treatment was on par with that from unweeded check. From these results, it is clear that application of flucetosulfuron at 10-12 and 18-20 DAS could significantly deplete soil weed seed bank compared to the other weed management practices adopted.

\section{Conclusion}

The total number of grasses, broadleaved weeds and sedges emerged at 14, 28, 42 and 56 DAl was significantly lower when flucetosulfuron was applied @ 20, 25 and $30 \mathrm{~g} \mathrm{ha}^{-1}$ at 10-12 and 1820 DAS compared to the application of herbicide at 2-3 DAS in the field experiment indicating its efficacy in depleting the weed seed bank in post experiment soil.

\section{REFERENCES}

1. Ambrosio, L.A., Iglesias, L., Marin, C., and Monte, J.P. (2004). Evaluation of sampling methods and assessment of the sample size to estimate the weed seed bank in soil, taking into account spatial variability. Weed Res, 44:224-236.

2. Anwar, M.P., Juraimi, A.S., Puteh, A., Man, A., and Rahman, M.M. (2012). Efficacy, phytotoxicity and economics of different herbicides in aerobic rice. Acta Agric. Scandl 62:604-615.

3. Barberi, P., and B. Lo Cascio. (2001). Long-term tillage and crop rotation effects on weed seed bank size and composition. Weed Res, 41:325-340. doi:10.1046/j.1365-3180.2001.00241.

4. Barberi, P., Cozzani, A., Macehia, M., and Bonari, E. (1998). Size and composition of the weed seed bank under different management systems for continuous maize cropping. Weed Res, 38:319-334.

5. Bhagat R.M., Bhuiyan S.I., and Moody K. (1999). Water, tillage and weed management options for wet seeded rice in the Philippines. Soil Tillage Res, 52:51 -58 .

6. Chauhan, B.S., Migo, T., Westerman, P.R., and Johnson, D.E. (2010). Post dispersal of weed seeds in rice fields. Weed Res, 50:553-560. http:// dx.doi.org/10.1111/j.1365-3180.2010.00807.

7. Feng, L., G.Q. Chen, X.S. Tian, H.M. Yang, M.F. Yue, and C.H. Yang. (2015). The hotter the weather, the greater the infestation of Portulaca oleracea: Opportunistic life-history traits in a serious weed. Weed Res, 55:396-405. doi:10.1111/wre.12151.

8. Hossain M.M., and BegumM. (2015). Soil weed seed bank: Importance and management for sustainable crop production- A Review.J. Bangladesh Agric. Univ., 13:221-228.

9. Hossain, M.M., Begum, M., Hashem, A, Rahman, 
Arya, S.R. and Syriac, E.K. / J. Appl. \& Nat. Sci. 10 (3): 853 - 859 (2018)

M.M., Bell, R.W., and Haque, M.E. (2014). Yield response of mustard as influenced by weed management practice under conservation agriculture system. Bangladesh J. Weed Sci., 4 and 5:87-92.

10.Hyvolen, T., and Salonen, J. (2012). Weed species diversity and community composition in cropping practices at two intensity levels-a six-year experiment. Plant Ecology, 159: 73-81.

11.Islam, Md.S. (2012). Effect of weeding regime on soil weed seed bank status and yield performance of transplanted Aman rice. MSc. (Ag) thesis, Bangladesh Agricultural University, Mymensingh, 53p.

12.Juraimi, A.S., Uddin, Md.K., Anwar, Md.P., Mohamed, M.T.M., Ismail, M.R., and Man, A. (2013). Sustainable weed management in direct seeded rice culture: A review. Aus. J. Crop Sci, 7(7): 989- 1002.

13.KAU [Kerala Agricultural University]. (2011). Package of Practices Recommendations: Crops (14 ${ }^{\text {th }} \mathrm{Ed}$.) Kerala Agricultural University, Thrissur, 360p.

14.Kamoshita, A., Ikeda, H., Yamagishi, J., Lor, B. and Ouk, M. (2016). Residual effects of cultivation methods on weed seed banks and weeds in Cambodia. Weed Biology and Management, 16: 93-107.

15.Luschei, E.C. (2003). Comparison of the effectiveness of seed bank sampling to seedling counts in reducing the uncertainty in estimates of weed population size. Aspects of Applied Biology, 69:137-142.

16.Menalled, F.D. 2013. Manage the Weed Seed Bank-Minimize "Deposits" and Maximize "Withdrawals". Available at: extension.org http:// www.extension.org/pages/18527/manage-the-weedseed bankminimize-deposits-andmaximizewithdrawals\#. VkG6 XI53C1t

17.Mesquita, M.L.R. 2017. Weed Seed bank in Rice Fields In: Advances in International Rice Research. http://www.intechopen.com/books/advances-in- international-riceresearch (accessed 2 April 2018)

18.Mohler, C.L., Dykeman, C., Nelson, E.B., and Ditommaso, A. (2012). Reduction in weed seedling emergence by pathogens following the incorporation of green crop residue. Weed Res, 52:467-477. http:// dx.doi.org/10.1111/j.1365-3180.2012.00940.x

19.Munhoz, C.B.R., Felfli J.M. 2006. Phytosociology of the herbaceous and sub-shrub layer of a savannah in Federal District, Brazil. Acta Botanica Brasilica. 20:671-685. http://dx. doi.org/10.1590/S0102 33062006000300017

20.Pereira, J.R., A.E., Duarte, J.B., Pitombeira, M.A.P., da Silva, N.E., de M. Beltrão, and L.M., Barros. (2013). Herbicide combinations to control the weed seed bank in an upland cotton field. Int. J. Experimental Bot. 82: 275-279

21.Singh, A., Kaur, R., Kang, J.S. and Singh, G. (2012).Weed dynamics in rice-wheat cropping system. Global J. Biology Agric. and Health Sci, 1(1):7-16

22.Teasdale, J.R., Magnum, R.W., Radhakrishnan, J., and Cavigelli, M.A. (2004). Weed seed bank dynamics in three organic farming crop rotations. Agron. J, 96: 1429-1435.

23.Vasileiadis, V.P., R.J., Froud-Williams, and I.G. Eleftherohorinos.(2007). Vertical distribution, size and composition of the weedseedbank under various tillage and herbicide treatmentsin a sequence of industrial crops. Weed Res, 47:222-230.doi:10.1111/ j.1365-3180.2007.00564.x

24.Walia, U.S., and L.S., Brar. 2006. Effect of tillage and weed management on seed bank of Phalaris minor Retz. in wheat under rice-wheat. Indian J. Weed Sci, 38:104-107.

25.Wilson, R.G., Kerr, E.D., and Nelson, L.A. 1985. Potential for using weed seed content in the soil to predict future weed problems. Weed Sci, 33:171-175. 